\title{
Evaluar para avanzar el sentido de la evaluación en el contexto académico
}

\author{
Nidia Stella Rincón Parra ${ }^{1}$
}

\begin{abstract}
Resumen
El presente es un ensayo argumentativo que pretende contextualizar la forma en que la comunidad científica-académica entiende la evaluación. Busca, a su vez, mostrar cómo ese proceso evaluativo debe encaminarse en las instituciones formadoras para que dichos procesos tengan sentido y significado. Se realiza un estudio comparativo del tratamiento que cuatro representantes de la comunidad científico-académica le dan a la evaluación a la hora de comprenderla. Metodológicamente se hace uso de un tipo de investigación documental descriptiva investigativa. Se tiene como población a cuatro $(30 \%)$ de los doce $(100 \%)$ autores más significativos y representativos de la comunidad científico-académica y se utiliza el análisis de contenido como técnica de recolección. Igualmente, se toman cuatro categorías de análisis como son la concepción, enfoques, importancia y aplicación de la evaluación en el contexto académico. El ensayo propone abordar la evaluación en forma balística, es decir, en todas las funciones que ella desempeña. Esto significa no quedarse solamente en lo referente al aprendizaje de los estudiantes sino hacer una introspección en la cultura de la evaluación como proceso para así mejorar la calidad de vida y la calidad de la educación. Esto contribuirá de manera integral en la formación del ser humano.
\end{abstract}

Palabras claves: Evaluación, sentido, significado, juicio de valor, construcción del conocimiento 


\title{
Evaluate to advance, meaning of evaluation in academic context
}

\begin{abstract}
Evaluate progress, the sense of evaluation in the academic context of distance education is an argumentative essay in which they seek to make a contextualization of what the scientific community academic assessment is understood by and in turn show how the evaluation process should be aimed at training institutions so that these processes have meaning and significance. We performed a comparative study of the treatment (4) four representatives of the scientific community - academic evaluation give to understand it. Methodologically, uses a type of descriptive research documentary research, having as a population of four $(30 \%)$ of the twelve $(100 \%)$ most significant and representative authors of the scientific community - academic, using content analysis as a technique for collecting and using four categories of analysis such as design, focus, relevance and application of evaluation in the academic context. The paper proposes to make the evaluation as ballistic, saying in all the functions it performs, this means not stay only in relation to student learning, but to an insight into the culture of evaluation as a process to improve quality of life and quality of education in a comprehensive manner contributing to the formation of human
\end{abstract}

Key words: Evaluation, meaning, significance, value judgments, knowledge construction

\section{lntroducción}

El mundo de la educación como el de la vida, está atravesado por los procesos evaluativos en todo momento. Tales procesos, vistos como posibilidades para entender y comprender lo que se hace, buscan ajustar nuestros rumbos y andares acorde con los horizontes que se han trazado. Por lo tanto, este ensayo argumentativo busca, por una parte, elaborar algunas conceptualizaciones sobre lo que la comunidad científica-académica está entendiendo por evaluación y, por otra, mostrar que ese proceso debe encargarse a instituciones formadoras 
para hacer que ellos tengan sentido y significado. Por último, se presentan algunas conclusiones que recogen el proceso anterior y que al mismo tiempo permitan vislumbrar nuevas posibilidades investigativas y de reflexión.

\section{Diferentes dimensiones del universo de la evaluación}

La evaluación puede ser abordada desde diferentes autores, cuyos matices y propuestas son disímiles. Este ensayo se centró en cuatro grandes autores que han hecho de la evaluación un espacio de indagación y cuyas propuestas tienen un gran significado para el desarrollo de la misma. Ellos son Jean-Marie Barbier, Robert McCormick, Ernest R. House y José Gimeno Sacristán, quienes en compañía de otros autores serán los que den las bases necesarias para las conceptualizaciones evaluativas que servirán de horizonte a la discusión en que se basa este escrito.

Partiendo de las anteriores consideraciones, Barbier (1999)entiende que, a partir del plano netamente semántico, el concepto de evaluación muestra una amplitud considerable. Se puede inclusive hablar de una inflación del concepto, pues se utiliza para designar el resultado de una acción de formación, la operación de selección humana que puede encerrar esta acción o simplemente lo que los participantes han podido pensar de las cualidades de su anfitrión..." (Barbier, 1999). De esta manera, él conduce a la siguiente definición:

[N]uestra propuesta lleva a considerar que existe control o seguimiento de la formación cada vez que se encontramos en presencia física o cognitiva de operaciones que sólo tienen aparentemente como resultado la emisión de informaciones acerca del funcionamiento concreto de una actividad de información. Existe, por el contrario, evaluación cada vez que nos encontramos ante operaciones que tienen como resultado la emisión de un juicio de valor sobre las actividades de formación (Barbier, 1999:-).

Para Barbier entonces, es el juicio de valor lo que le da sentido y dirección al proceso evaluativo. Esto, como es natural, está antecedido de unos procesos que tienen que ver con la medición y con la recogida de los datos, pero estos momentos no constituyen lo que se puede denominar como evaluación, aunque sí los presupongan. Él establece esta distinción en los siguientes términos:

[C]omo vemos, la medida no implica evaluación; su aceptación es más limitada ("medir no es evaluar"), es una operación que permanece interna a la constitución de los datos de referencia: de hecho, no es más que una forma de tratamiento de la información obtenida que facilita manipulaciones, comparaciones y aplicaciones. La evaluación no implica medida, las informaciones "útiles" pueden continuar siendo de tipo cualitativo, como ocurre frecuentemente en el caso de la evaluación de acciones o con la heteroevaluación; por otra parte, es raro que en 
formación se puede limitar a una o a algunas variables. Efectivamente, la medida no es más que una operación posible en la constitución de los datos de referencia (Barbier, 1999:71).

Lo anterior está rompiendo con prácticas que tienen mucha sedimentación en las instituciones escolares -incluso en las universidades- como es el hecho de pensar que la evaluación se agota en la medición, expresada en una calificación. Esto está bien alejado de la realidad evaluativa. En esta dirección se orienta Cayetano Estévez Solano (2008), cuando concibe que la evaluación no es calificar comportamiento, no es examen o prueba, ni tampoco medición de conocimientos; por el contrario, ella está ligada a la valoración, a la apreciación y al análisis contextual de todo un proceso de experiencias previas.Es toda una praxis que permite llegar a una evaluación integral y que evidencia el aprendizaje significativo.

Ahora bien, las anteriores conceptualizaciones pueden ser comparadas con aquellas planteadas por McCormick y James), quienes expresan que:

[L]a evaluación en el contexto del desarrollo profesional y de la mejora de la enseñanza hace referencia a la revisión de la práctica con el fin de diagnosticar problemas, desarrollar, implementar y evaluar soluciones o, en caso contrario, asegurarse de que el proceso se desarrollan en forma correcta. Se da por supuesto que la evaluación de las prácticas concretas en contextos determinados es más importante en relación con las necesidades educativas y profesionales de los docentes y escuelas y, por tanto, más apropiadas para producir mejoras (1997:55).

Como se puede apreciar, estos autores resaltan la importancia de la evaluación a la hora de buscar el mejoramiento del proceso y no solo de los resultados. La evaluación quiere saber qué tan lejos o qué tan cerca se encuentra el logro del conocimiento y qué se busca para poder tener indicadores en los aspectos que deben mejorarse. Esto, a su vez, está ligado a un mecanismo de rendición de cuentas, ya sea en forma individual o en propuestas sociales. Rendir cuentas es mostrar a la sociedad que las inversiones que se hacen en educación tienen sus frutos concomitantes. Evaluar es entonces, desde esta perspectiva, un espacio que busca empoderar, potenciar, hacer que las cosas y los acontecimientos se cualifiquen, rompiendo así con las esquematizaciones y con la rigidez que a veces el quehacer educativo conlleva.

De la misma manera, para House (2002) la evaluación supone -por naturalezaadoptar un conjunto de normas, definirlas, especificar la clase de comparación y deducir en qué grado el objeto satisface las normas. Cumplimentadas estas etapas, el evaluador debe poder llegar a establecer un juicio sobre el valor del objeto evaluado. Otra vez se encuentra la evaluación ligada con la valoración que se hace sobre algo, o sobre un proceso. Ella, de nuevo, es la encargada de emitir un juicio; es la que permite trazar caminos, orientar rutas y permitir los 
avances. Así pues, una evaluación delinea, suministra información, obtiene y hace análisis sistemático de los datos para determinar el valor que encierra un proceso. Al permitir la emisión de un juicio, la evaluación da elementos valiosos para la toma de decisiones. Todos estos aspectos están enmarcados en las concepciones que se vienen presentando.

Para José Jimeno Sacristán (2009), la evaluación está incrustada en una malla compleja de interrelaciones. Al respecto dice: La práctica de la evaluación se explica por la forma en que se lleva a cabo las funciones que desempeña la institución escolar y por eso viene condicionada su realización por numerosos aspectos y elementos personales, sociales e institucionales; al mismo tiempo, ella incide sobre todos los demás elementos implicados en la escolarización: transmisión de conocimientos, relaciones entre tutores y aprendientes entre profesores y alumnos interacciones en el grupo, métodos que se practican, disciplina, expectativas de alumnos - profesores, para el caso de la Universidad Nacional Abierta y a Distancia UNAD tutores - aprehendientes, valoración del individuo en la sociedad, entre otros, ayuda decisivamente a configurar el ambiente educativo. Estudiar la evaluación es entrar en el análisis de toda la pedagogía que se practica.

La propuesta que se acaba de señalar, pone a la evaluación como algo nodal en quehacer pedagógico. Ella es reflexión y análisis para mejorar las prácticas y los procesos pedagógicos. Es comprobación de la eficacia de lo que se hace. Es desentrañar el significado de las prácticas educativas en el contexto de las instituciones. De tal modo, ante la pregunta ¿qué se entiende por evaluar?, Jimeno responde:

[E]evaluar hace referencia a cualquier proceso por medio del que alguna o varias características de un estudiante de un grupo de estudiantes, de un ambiente educativo, de objetivos educativos, de materiales, tutores y programas reciben en la atención del que evalúa, se analizan y se valoran sus características y condiciones en función de unos criterios o puntos de referencia para emitir un juicio que sea relevante para la educación (2009:-).

El elemento central y constante que se encuentra en todos los autores que se vienen analizando, es que la evaluación está referida a la emisión de juicios frente a la valoración de algo o de algún proceso. Pero esto no siempre fue así: a ello se llegó a través de proceso histórico. Haciendo referencia a la evaluación escolar, Jimeno hace la siguiente apreciación:

La evaluación de estudiantes se entendería desde esta óptica como el proceso por medio del cual los profesores, en tanto que son ellos quienes la realizan, buscan y usan información procedente de numerosas fuentes para llegar a un juicio de valor sobre el alumno en general o sobre alguna faceta particular del mismo (2009: 343). 
De esta manera, el concepto de «juicio» tiene una dimensión que va más allá de lo que se puede concebir como nota o como calificación. Pero el sentido de la evaluación supera las anteriores denominaciones sin negarlas, ya que las considera como una etapa necesaria de la misma. Por lo tanto, la evaluación también tiene que ver con las técnicas, con lo ético y con los procesos de comunicación. El universo de la misma tiene que dar cuenta del qué, del cómo, del por qué y del para qué se evalúa.

Son aquellas dimensiones las que hace que la evaluación sea un proceso complejo y de proporciones significativas. Jimeno (2009), haciendo eco a la propuesta de Stufflebeam, propone unos interrogantes que conllevan todo un horizonte de búsqueda para la comprensión de los procesos evaluativos. Esas preguntas son: ¿cómo definir la evaluación?, ¿cuáles son sus funciones?, ¿qué son objetos de evaluación?, ¿qué tipo de información exige la evaluación de algo en particular?, ¿qué criterio se tiene para decidir el método o la importancia de lo que es evaluado?, ¿a quién debe servir o a quién deben ser útiles los juicios de la evaluación?¿qué proceso hay que seguir para realizarla?, ¿qué métodos de indagación han de seguirse al evaluar?, ¿quién debe realizarla?, ¿con qué criterios ha de juzgarse la evaluación: por su utilidad, factibilidad, por criterios éticos, por su precisión?

Desarrollar todos los anteriores interrogantes está muy lejos de la pretensión de este ensayo, pero es importante dejar el camino abierto para la indagación y la búsqueda; con el convencimiento de que quienes quieran tomar esta ruta, allí encontrarán un acicate promisorio que los orientará.

\section{Enfoques de la evaluación}

Cuando se habla de enfoques se está haciendo referencia a los diferentes esquemas o características propias desde donde se está mirando y se realizan las diferentes prácticas evaluativas. De esta manera, en el decurso histórico se han encontrado diversos enfoques para pensar y llevar a cabo los procesos evaluativos. Uno de ellos hace énfasis en los procesos de medición, los cuales están cargado de estadísticas descriptivas que siguen ciertos valores numéricos. Aquí hay toma estandarizada de datos, escalas y correlatos que responden a una postura positivista en donde sólo es válido lo que se pueda medir y cuantificar. Pero hay otro enfoque que, teniendo como fundamento epistemológico al racionalismo, enfatiza una dimensión cualitativa. Aquí se resalta la subjetividad, se rescata la condición individual de los seres humanos y se les aborda como seres participativos, holísticos, sensibles y con capacidad para interpretar. Sin caer en una posición maniquea, es conveniente resaltar que los anteriores enfoques no pueden verse como algo separado en estancos. Por el contrario, lo que se busca es el debate y la convergencia para que la evaluación sea un proceso argumentativo que permita juzgar desde nuestras pertenencias; es decir, con lo que se tiene en nuestras mentes y a partir de aquello que somos. Esto, sin duda, ayuda a valorar y a emitir juicios concomitantes. 
Acorde con lo anterior, los personajes que se vienen analizando como representantes de la comunidad científica-académica sobre la evaluación, toman diversas posturas sobre los enfoques en evaluación. Por ejemplo, Barbier (1999) es partidario de un enfoque cualitativo. Él señala con toda claridad que la evaluación no puede ser cuantitativa: ella está relacionado con el juicio de valor y con la cualidad que lo sustenta; por lo tanto, la presencia de aquel juicio es lo que permite decir si hay o no evaluación. El juicio de valor constituye la señal de la evaluación (Barbier, 1999).

McCormick y James (1997) se inclinan por un enfoque que rescate lo colaborativo. Defienden la probabilidad de que los resultados de la evaluación lleven al perfeccionamiento de la educación. Para ellos, el perfeccionamiento será mayor si todos los que desempeñan alguna función práctica (especialmente los profesores) participan de manera activa en la identificación de aspectos que se deben evaluar, deciden estrategias generales, recogen y procesan información importante, proponen opciones normativas y deciden y desarrollan acciones. En otras palabras, la participación en el proceso de evaluación, hetero-evaluación y la co-evaluación reviste especial importancia.

House (2002) por su parte, n consecuencia con sus planteamientos éticos, señala que todos los enfoques evaluativos pueden tener validez. Agrega,

No cabe duda de que una evaluación no veraz no sea válida, y la verdad pueda establecerse de muchos modos. La postura positivista equipara la veracidad con la réplica y la predicción. Si conocemos la causa de algo, podemos observarlo y preverlo con exactitud; sin embargo, la veracidad abarca mucho más de lo que reconoce la concepción positivista. Los criterios de verdad dependen del acuerdo intersubjetivo de la comunidad interesada y cambian con el tiempo. En la situación individual, personal, se puede imaginar la búsqueda de la verdad como empeño individual, aunque no sea así (House, 2002:-).

Entonces, el enfoque más propicio para los anteriores autores es el que está matizado con los diferentes criterios de las personas que participan en el proceso. Esto lleva a pensar que ningún enfoque de evaluación, ningún método particular, garantizará de antemano la validez. Se tiene que mirar hacia atrás y examinar la evaluación concreta en su situación para ver si es válida. En la mayoría de los casos, resultarán adecuados diversos enfoques de evaluación y el evaluador puede escoger alguno de ellos dependiendo de sus propias preferencias, de su contexto y del proceso llevado a cabo. Muchas evaluaciones serán mezclas de varios enfoques.

Por otra parte, el enfoque que defienden Gimeno Sacristán y Pérez Gómez (1993) responde a la necesidad de integrar el proceso del aprender y del enseñar. Para esto es necesario reclamar la evaluación integrada en el proceso 
de enseñanza-aprendizaje; una exigencia pedagógica que no es fácil satisfacer, pues se precisan unas condiciones de partida. Estas condiciones están relacionadas con la finalidad de obtener información que no sea distorsionada para así lograr un mejor conocimiento de los alumnos y permitir un clima placentero de relaciones humanas.

Para que lo anterior sea posible, Gimeno Sacristán y Pérez Gómez (1993) proponen el siguiente mecanismo: el procedimiento de hacer posible la evaluación integrada es plantearla de manera interactiva, es decir durante el proceso de enseñanza-aprendizaje. Es preciso entenderla como un proceso natural de información sobre lo que ocurre, que utiliza múltiples recursos, sin plantear necesariamente procedimientos formales de evaluar. Se trata de conocer al estudiante y se precisa de una atención consciente y reflexiva por parte de los profesores (como una preocupación más de éstos cuando enseñan). Más que pensarla como algo que se sirve de procedimientos especiales, la evaluación es una actividad que descansa en las capacidades del profesor (naturales y adquiridas por formación) para comprender situaciones, reacciones de los alumnos, rasgos significativos de cómo ejecutar las tareas, el nivel de sus realizaciones, de las dificultades que van encontrando y del esfuerzo que ponen.

Como se puede apreciar, cada uno de los autores que se vienen analizando remarcan varios enfoques frente a la evaluación. Pero lo más importante es que ella, sea cual fuere el enfoque con que se la mire, tiene una importancia significativa para la educación, para la sociedad y para la cultura en general.

\section{Importancia de la evaluación}

Para todos los autores que se vienen señalando, la evaluación se presenta como un proceso de gran importancia, tanto para la vida académica como para el contexto socio--cultural. Esa relevancia está determinada por la función que ésta desempeña en los aquellos ámbitos (académico y sociocultural), así como por el objeto concomitante con su naturaleza. De esta manera, la información que ella produce es el elemento clave que permite la ponderación y los juicios de valores.

Para Barbier (1999) por ejemplo, la evaluación, como disciplina y como práctica profesional, tiene su importancia en la medida en que da respuestas objetivas y fiables a las diferentes interrogantes que tienen que ver con el desarrollo, con la maximización de las ventajas y con la minimización de los inconvenientes. House (2002) a su vez, recalca la necesidad de debatir la ligazón entre evaluación y poder, lo cual está ceñido a la perspectiva de contextos que faciliten los dominios pertinentes que los individuos tienen que operar en los espacios sociales. McCormick y James (1997) por su parte, están empeñados en ver la importancia de la evaluación para proporcionar 
diversos tipos de información a los grupos responsables de tomar decisiones, entre ellos los profesores, padres, govemors, funcionarios y políticos". Gimeno Sacristán y Pérez Gómez (1993) no dejan de pensar la evaluación como capacidades del docente (naturales y adquiridas por formación) para comprender situaciones, reacciones de los estudiantes, rasgos significativos de cómo ejecutan las tareas, nivel de sus realizaciones, de las dificultades que van encontrando, del esfuerzo que ponen.

\section{Balances finales y perspectivas sobre la evaluación en Educación a distancia}

La preocupación por el sentido de la evaluación ha estado y está presente en el proceso educativo nacional así como en el contexto universal y regional de América Latina. En Colombia hay un sentimiento de que el sistema educativo no funciona de modo eficaz, eficiente y con equidad para todos los componentes de la comunidad académica. Esta preocupación cobra especial importancia en los momentos actuales: una época de grandes transformaciones en el cual el mundo moderno está requiriendo de la universidad nuevos comportamientos que vayan más allá de las repeticiones de discursos previamente elaborados, y que se inscriba, más bien, en la búsqueda de conocimientos, en el razonamiento crítico y en la identificación y solución de problemas. En este contexto la evaluación toma nuevas dimensiones y necesita de nuevas formas de abordar los conocimientos.

Aquí se está pidiendo una evaluación que se pregunte cómo los estudiantes están aprendiendo, cómo están desarrollando la capacidad para entender el mundo y cómo ese saber puede impactarlo positivamente (a él/ella y a la vez a su entorno). En este sentido, lo que se busca es que los individuos adquieran las competencias necesarias para abordar la realidad en forma flexible; es decir, que la investigación, la imaginación, la percepción del mundo y los procesos creativos a través de las TICs sean utilizados como dinamizadores en el proceso de construcción del conocimiento significativo.

El papel de la evaluación, acorde con todos los autores mencionados, tiene un gran papel: informar sobre los aspectos más importantes que se requieren para comprender el mundo en el aprendiente y de esta forma participar en la construcción de la sociedad. La evaluación en el marco que se acaba de señalar, le proporciona a la educación a distancia elementos indispensables para su mejora cualitativa. Pero ¿cuáles serían esos aportes que la evaluación le proporcionaría a la educación para su desarrollo?

Recogiendo todas las argumentaciones que han proporcionado los diferentes autores, se puede remarcar los siguientes aspectos: primero, la evaluación proporciona a la educación datos, análisis y valoraciones pertinentes para que ella pueda tener un conocimiento valido de lo que acontece en su dinámica interna. En otras palabras, ella realiza una función diagnóstica que indefectiblemente va 
a permitir una toma de decisiones de la forma más adecuada y científicamente elaborada. Esto, en el campo del aprendizaje de los estudiantes y en todos los aspectos que tienen que ver con el desarrollo educativo.

Un segundo aspecto que la evaluación proporciona al quehacer educativo está orientado hacia los procesos de cambio. Al decir dónde se encuentran, estos procesos son elementos indispensables para orientar la búsqueda en forma intencional. Cambiar significa plantearse metas y trabajar por alcanzarlas así que la evaluación, por consiguiente, puede contribuir con sus aportes de sistematización y rigurosidad a los procesos de cambio. Pero más allá de los procesos que se han venido señalando, la evaluación tiene una tercera dimensión que está orientada hacia la valoración de los resultados. Tales resultados tienen que ver con diversos aspectos tales como competencias para el trabajo, métodos rigurosos, valores ciudadanos, reducción de las desigualdades sociales, entre otros.

Hay un cuarto aspecto que la evaluación cumple como función indispensable para mejorar la educación: fundamentar un área de la calidad; es decir, contribuir a la mejora de organizaciones educativas. La evaluación, por consiguiente, orienta su quehacer hacia el mejoramiento de los métodos didácticos, de las relaciones interindividuales y grupales, del clima académico, de la distribución y utilización de los recursos y del desarrollo del currículo -por no mencionar sino los más significativos. Esta mirada micro de las organizaciones educativas encuentra en la evaluación un elemento indispensable para su mejora cualitativa, ya que permite iluminar la situación general y de esa forma permite construir nuevas dinámicas de calidad que deben desarrollarse.

\section{Conclusiones}

Todo el recorrido que se ha realizado en diálogo con la comunidad científica y académica sobre la evaluación (especificada en los autores que se han tomado como referencia) conduce a la comprensión de que ella cumple una función indispensable en la vida social en general y en los aspectos educativos en particular.

La evaluación debe tomarse en forma holística, es decir en todas las funciones que ella desempeña. Esto significa no quedarse solamente en lo referente al aprendizaje de los estudiantes, sino entrarse en la cultura de la evaluación como proceso para mejorar la calidad de vida y la calidad de la educación; pero al mismo tiempo, es necesario hacer una evaluación de la evaluación que se realiza en las organizaciones educativas. Esta metaevaluación, indefectiblemente, conducirá a una cualificación de los proceso evaluativos. 


\section{Referencias Bibliográficas}

Barbier, J.M. 1999. La evaluación en los procesos de formación. Barcelona: Paidós.

Binet, A. 1929. La psicología del razonamiento: investigaciones experimentales por el hipnotismo. Madrid: Biblioteca Científico-Filosófica.

Estévez Solano, C. [1996] 2008. Evaluación integral por procesos. Una experiencia construida desde el aula. Bogotá: Cooperativa Editorial Magisterio.

Gimeno Sacristán J. y A.I. Pérez Gómez. 1993. Comprender y transformar la enseñanza. Madrid: Ediciones Morata.

House, E. R. 2002. Evaluación, ética y poder. Madrid: Ediciones Morata.

McCormick, R y M. James. 1997. Evaluación del curriculum en los centros escolares. Madrid: Ediciones Morata. 